\title{
Finite-element based electromagnetic field simulations: Benchmark results for isolated structures
}

\author{
Sven Burger, ${ }^{a b}$ Lin Zschiedrich, ${ }^{a}$ Jan Pomplun, ${ }^{a}$ Frank Schmidt, ${ }^{a b}$ \\ a JCMwave GmbH, Bolivarallee 22, D - 14050 Berlin, Germany \\ ${ }^{b}$ Zuse Institute Berlin (ZIB), Takustraße 7, D-14 195 Berlin, Germany
}

This paper will be published in Proc. SPIE Vol. 8880 (2013) 88801Z, (Photomask Technology 2013, DOI: $10.1117 / 12.2026213)$, and is made available as an electronic preprint with permission of SPIE. One print or electronic copy may be made for personal use only. Systematic or multiple reproduction, distribution to multiple locations via electronic or other means, duplication of any material in this paper for a fee or for commercial purposes, or modification of the content of the paper are prohibited.

\begin{abstract}
We use a finite-element method to obtain highly converged results for a nano-optical light scattering setup with a non-periodic geometry.
\end{abstract}

Keywords: 3D rigorous electromagnetic field simulation, non-periodic pattern, finite-element method, computational metrology, computational lithography

\section{INTRODUCTION}

Electromagnetic field (EMF) simulations are important for design of experimental setups and understanding of experimental results in science and technology. As prominent example, recently in nanoscience and -technology and in related semiconductor industries, efficient (= precise $\&$ fast) EMF simulations are given significant attention! 1 Performance of lithography at deep ultraviolet (DUV) and extreme ultraviolet (EUV) wavelengths can be pushed using computational methods. ${ }^{2}$ Further, computational methods are an integral part of optical metrology setups in this field ${ }^{3}$ EMF simulation of non-periodic patterns at high numerical resolution is gaining importance for metrology applications like in-die photomask registration and metrology and critical-dimension (CD) metrology and for many lithography applications handling finite patterns or isolated assist features.

Several numerical methods are used for EMF simulations, including, e.g., finite-difference time-domain methods (FDTD), rigorous coupled wave analysis (RCWA) and finite-element methods (FEM)!4 This paper concentrates on FEM in an implementation by JCMwave. ${ }^{5}$ In previous contributions these finite-element methods have been applied to various scientific and technological fields. 6 These include also computational lithography tasks like investigations of 3D effects, source-mask optimization, analysis of the impact of line edge/width roughness and defects, as well as to computational metrology tasks like cricital dimension (CD) metrology at EUV wavelengths and metrology of 3D patterns. ${ }^{7}[11$

In order to demonstrate FEM performance for simulation of non-periodic patterns, in this contribution we revisit a specific benchmark example for electromagnetic field solvers. The benchmark setup consists of computing the near field in an isolated (i.e., non-periodic) pattern illuminated by a plane wave. We demonstrate numerical convergence of our method with various numerical parameters, in quantitative agreement with results from the literature ${ }^{12}$ and we demonstrate an advance in performance by several orders of magnitude when compared to results from the literature $!^{12}$ Previous benchmarks including this solver mainly concentrated on periodic patterns and other devices. 13,16

Corresponding author: S. Burger

URL: http://www.zib.de

URL: http://www.jcmwave.com 

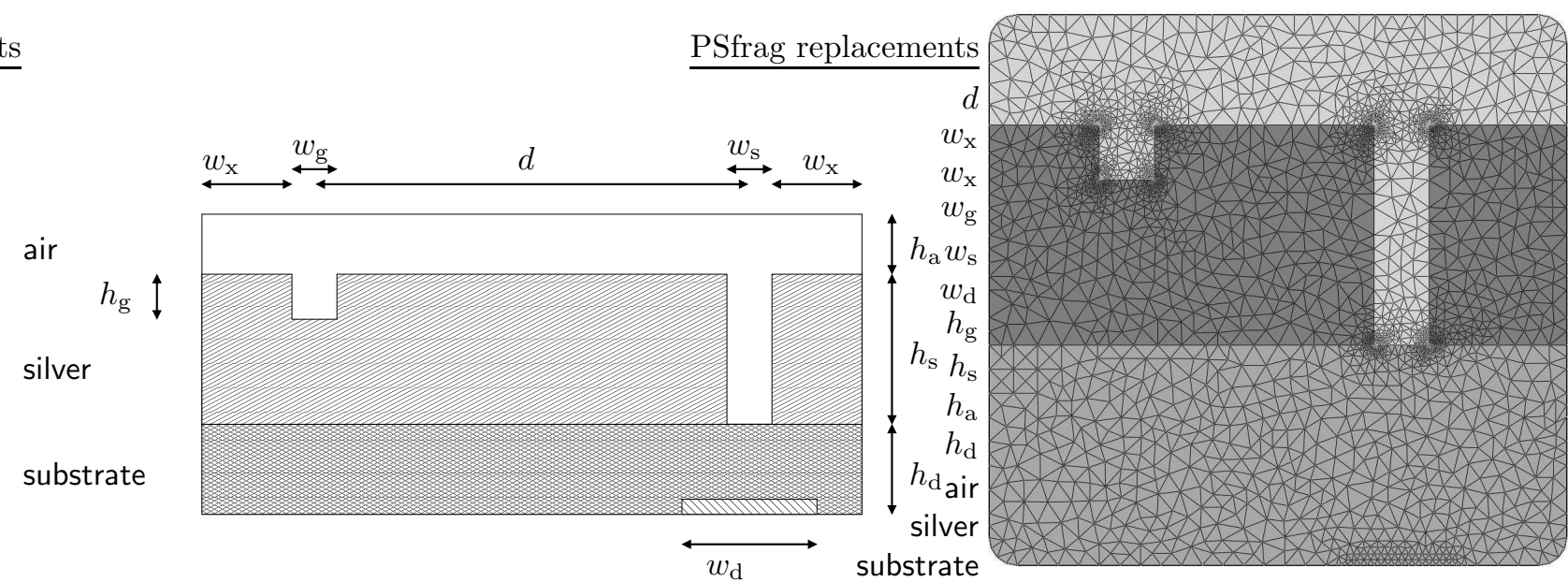

Figure 1. Left: Schematics of the geometry of a slit and a groove in a silver layer on a substrate. At the left and right boundary of the depicted geometry, the layer stack of substrate, silver film and air is extended to infinity. The physical parameter values (geometrical parameters $w_{\mathrm{g}}, w_{\mathrm{s}}, w_{\mathrm{d}}, h_{\mathrm{g}}, h_{\mathrm{s}}, h_{\mathrm{d}}$ material and source parameters) are given in Table 1 The distances of the computational domain boundary from the scattering slit and groove, $w_{\mathrm{x}}$ and $h_{\mathrm{a}}$, are numerical parameters which are varied in the numerical tests, cf. Table 3 . Right: Visualization of a mesh discretizing the geometry of the slit-groove setup. Note the finer discretization at the corners of the slit and of the groove cross-sections.

This paper is structured as follows: The benchmark setup is described in Section 2 the numerical method and obtained results are presented in Section 3. Selected numerical values obtained in the convergence studies are tabulated in the Appendix.

\section{BENCHMARK: NON-PERIODIC DIFFRACTION PROBLEM}

We revisit a numerical benchmark problem for an isolated (non-periodic) setup which has been described by Lalanne et al.12] The problem models an isolated, sub-wavelength slit in a silver film on a substrate with a neighboring, parallel groove in the silver film. This setup is illuminated by a plane wave at perpendicular incidence from above and with in-plane electric field polarization (resp. out-of-plane magnetic field polarization). The energy flux of light, $S$, transmitted through the slit to a detector region of width $w_{\mathrm{d}}$, placed a distance $h_{\mathrm{d}}$ below the slit is detected and normalized to the energy flux $S_{0}$ through the slit, computed in a second simulation where the groove is not present.

Due to the geometrical, source and material properties plasmonic effects lead to a very critical dependence of normalized transmission $S / S_{0}$ on the physical parameters. In essence, the source field is scattered at the groove and slit structures. This excites highly peaked surface fields which propagate along the surface, interfere and are re-scattered at the slit and groove structures. The relatively simple physical setup combined with the critical electromagnetic behavior makes this configuration a very interesting benchmark case. The choice of the geometry was originally also motivated by experimental results 12

\begin{tabular}{|l|l||l|l|}
\hline$d$ & $500 \mathrm{~nm}$ & $\lambda_{0}$ & $852 \mathrm{~nm}$ \\
$w_{\mathrm{s}}=w_{\mathrm{g}}$ & $100 \mathrm{~nm}$ & & \\
$w_{\mathrm{d}}$ & $200 \mathrm{~nm}$ & $\varepsilon_{\mathrm{Ag}}$ & $-33.22+1.170 \mathrm{i}$ \\
$h_{\mathrm{g}}$ & $100 \mathrm{~nm}$ & $\varepsilon_{\text {substrate }}$ & 2.25 \\
$h_{\mathrm{s}}=h_{\mathrm{d}}$ & $400 \mathrm{~nm}$ & $\varepsilon_{\text {air }}$ & 1 \\
\hline
\end{tabular}

Table 1. Physical parameter setting for the benchmark simulations. Dimensional parameters (compare Fig. 1): slit-groove distance, $d$, slit and groove widths, $w_{\mathrm{s}}$ and $w_{\mathrm{g}}$, detector width, $w_{\mathrm{d}}$, slit and groove height, $h_{\mathrm{s}}, h_{\mathrm{g}}$, and detector distance from slit, $h_{\mathrm{d}}$. Source vacuum wavelength $\lambda_{0}$ and complex material relative permittivities $\varepsilon$. 
The geometry of the slit-groove configuration is depicted schematically in Fig. 1. The corresponding parameters, and the material and source properties are given in Table 1.

In the original benchmark publication $\frac{12}{12}$ different numerical methods and implementations are used to obtain twelve different numerical results. The average result is $S / S_{0} \approx 2.18$ with a standard deviation of about 0.03 (cf. Fig. 5 in ${ }^{12}$ ). Internal convergence (i.e., convergence toward a specific quasi-exact result for each method) is observed with all methods. However, different levels of accuracy are reached, best internal relative accuracies are up to about $10^{-5}$ which is significantly lower than the deviations of the best converged results of the different methods from each other. Table 2 summarizes some of these results.

\section{NUMERICAL METHOD AND RESULTS}

\subsection{Finite element method}

For rigorous simulations of the electromagnetic near field we use the FEM Maxwell solver JCMsuite. This solver includes implementations of higher-order edge-elements, self-adaptive meshing, and fast solution algorithms for solving time-harmonic Maxwell's equations! [5] [18

Briefly, the simulations are performed as follows: a scripting language (Matlab) automatically iterates the numerical input parameters for fixed physical project parameters. The build-in mesh generator triangulates the geometries with and without the groove. Figure1 (right) shows a graphical representation of a mesh. The FEM solver computes the near fields, and in a post-process computes from these the integrals over the electromagnetic field energy flux densities (Poynting vector field) in the detection region, $S$ and $S_{0}$.

Numerical parameters in the simulation setup are the polynomial degree of the finite-element ansatz functions, $p$, the number of (adaptive or global) grid refinement steps, $n_{\text {adapt }}$, resp. $n_{\text {reg }}$, the offset of the structures in the computational domain from the boundaries of the computational domain, $w_{\mathrm{x}}$ (with $h_{\mathrm{a}}=w_{\mathrm{x}}$ ), a minimum mesh size for local prerefinement of the initial meshes around corners of the geometry, $d x$, and a sidelength-constraint, $S L C$, defining the maximum sidelength of the triangles of the initial mesh (prior to the $n_{\text {adapt }}+n_{\text {reg }}$ mesh refinement steps). Discretizing Maxwell's equations for given numerical parameters yields a linear system of equations with $N$ unknowns. Typically, when only one numerical parameter is varied, an increase of $N$ yields an increase of accuracy of the discrete solution, at least when a convergent regime is reached. In our case we have several parameters at hand, $p, n_{\text {adapt }}, n_{\text {reg }}, w_{\mathrm{x}}, d x, S L C$. All of these influence $N$, but influence of these parameters on the accuracy of the discrete solution should be quite different: Increase of FEM degree $p$ is expected to greatly improve accuracy at significantly increased numerical costs, $N$. For field distributions with
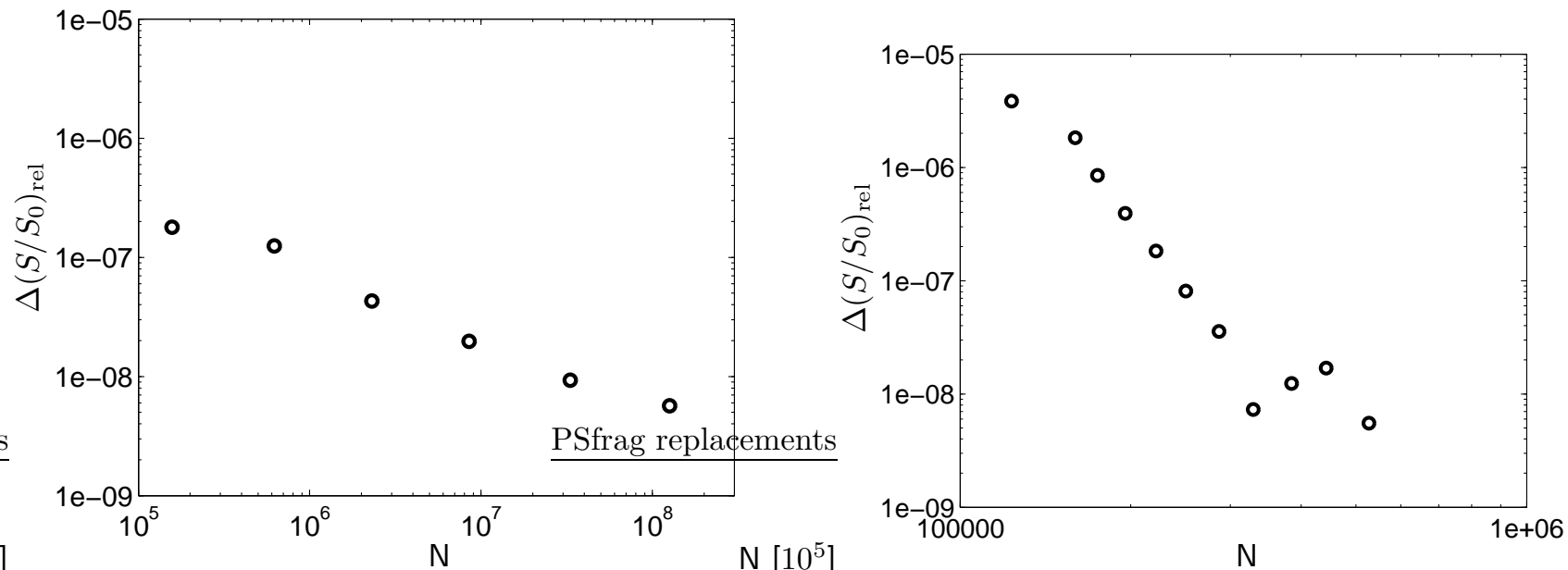

Figure 2. Convergence: Dependence of the relative numerical error, $\Delta\left(S / S_{0}\right)_{\text {rel }}$, on the number of unknowns in the FEM problem, $N$. The data points are generated using regular mesh refinement (left) and an automatic, adaptive mesh refinement strategy (right). Finite elements of polynomial order $p=4$ have been used, compare also Table 3 
highly localized field peaks (as is present at the given metal edges and corners) accuracy improvement through local mesh refinement, influenced by parameters $n_{\text {adapt }}$ and $d x$ is expected to be dominant at relatively low impact on $N$, compared to regular mesh refinement, controlled by parameters $n_{\text {reg }}$ and $S L C$. For the computational domain offset, no influence on the accuracy is expected as long as the boundary conditions are implemented as reflection-free boundaries (transparent boundaries, relatized by so-called perfectly matched layers, $\mathrm{PML}^{18}$ ).

For each set of numerical parameters, the result $S / S_{0}$ is saved, together with the number of unknowns $N$ and the total CPU time for computing $S$ and $S_{0}$. Computations are performed on a standard computer with extended RAM (necessary for computations with high $N$ ).

\subsection{Numerical results}

FEM computations using JCMsuite yield a result of $S / S_{0} \approx 2.19882594$. In order to estimate the numerical error of our computed result and in order to show that the result does not depend on possible systematic errors introduced by the method we have performed computations for a variety of numerical parameter sets. Some of the results are listed in the Appendix, in Table 3. As no reliable reference value at sufficient accuracy is at hand we use the result of $S / S_{0}$ for the numerical parameter data set with highest finite element degree $p=9$ and rather fine meshing as quasi-exact result, $S / S_{0, \mathrm{qe}}$. The relative error of a numerical result for $S / S_{0}$ is then defined as $\Delta\left(S / S_{0}\right)_{\text {rel }}=\left|S / S_{0}-S / S_{0, \mathrm{qe}}\right| /\left(S / S_{0, \mathrm{qe}}\right)$.

Convergence with mesh refinement Figure 2 shows how the relative error decreases with increase in computational effort, reached by regular mesh refinement (Fig. 2, left) and adaptive mesh refinement (Fig. 2. right). For adaptive mesh refinement, a local error-estimator checks locally smoothness of the solution and yields local mesh refinement for only those elements with relatively high estimated errors. For regular mesh refinement, all triangles are refined to four smaller triangles, each. Both methods yield highly accurate results, however for adaptive mesh refinement computational costs are greatly reduced when compared to global mesh refinement. The polynomial degree of the finite element ansatz functions (FEM degree) was fixed to $p=4$ in these simulations (compare the first two data sets in Table 3).

Convergence with FEM degree Convergence of numerical error with FEM degree is displayed in Figure 3 (left). For these simulations the mesh refinement strategy was fixed to always the same initial mesh and three adaptive refinement steps. FEM degree $p$ is varied between $p=1$ and $p=7$. For $p>4$ a relative error below $10^{-9}$ is reached, $\Delta\left(S / S_{0}\right)_{\text {rel }}<10^{-9}$.

Convergence with local prerefinement Figure 3 (right) shows convergence of the numerical error when both, $p$ and mesh refinement strategy are fixed, but the initial mesh is locally prerefined around the slit and
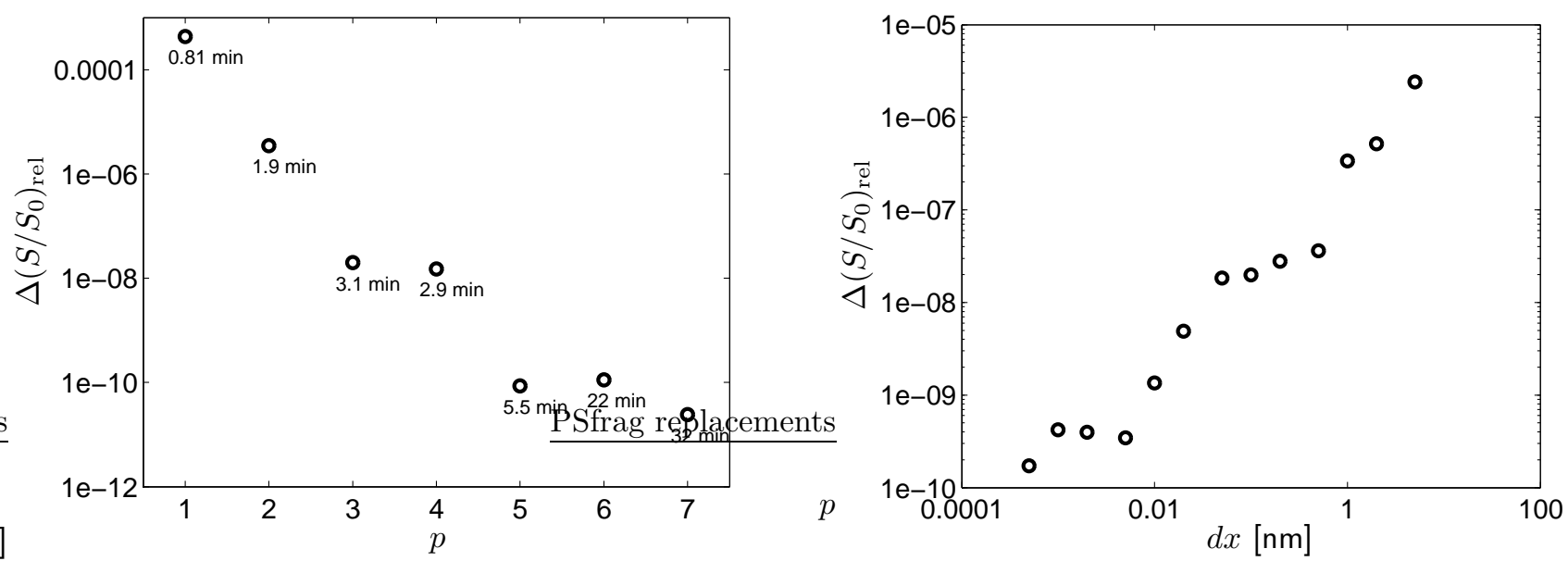

Figure 3. Convergence: Left: Dependence of the relative numerical error, $\Delta\left(S / S_{0}\right)_{\text {rel }}$, on the polynomial order of the finite element ansatz functions, $p$ for $n_{\text {adapt }}=1$ and $n_{\text {reg }}=2$ mesh refinement steps. Total computation times on a standard PC are indicated. Right: Dependence of the relative numerical error, $\Delta\left(S / S_{0}\right)_{\text {rel }}$, on the minimum mesh size at around the corners of the edges of the slit and of the groove, $d x$. Please, compare also Table 3 
groove edges with local mesh fineness $d x$. Numerical accuracy shows a strong dependence on this parameter. Relative numerical errors below $10^{-9}$ are reached for $d x<0.01 \mathrm{~nm}$ (using FEM degree $p=7$, three regular mesh refinement steps and two adaptive mesh refinement steps). Very good local accuracy at critical positions in the near field obviously has a great impact on the accuracy of energy flux obtained at the detector position. This observation is similar to the observation of the advantage of adaptive mesh refinement versus global mesh refinement. It might also explain the relatively large error ranges and absolute deviations of the different methods highlighted in the literature $\frac{12}{2}$ since here mainly non-adaptive methods have been used or adaptivity was restricted to relatively coarse sampling (in the case of Fourier modal methods with complex coordinate transforms, MM3 $3^{12}$ ).

Negligible influence of domain boundary placement and initial meshing Finally Figure 4 shows that neither the global initial mesh refinement, $S L C$ (Fig. 4 left), nor the distance of the computational domain boundary from the scattering structures, $d x$ (Fig. 4. right) has influence on the computational results larger than the error ranges which can be expected from used mesh refinement and finite element polynomial degree parameters, $n_{\text {adapt }}+n_{\text {reg }}, d x$, and $p$. The fact that the computational domain boundary placement has no effect on the numerical error strongly indicates that influences of numerical parameters for realizing transparent boundary conditions are not present in this case.

Further tests We have tested that using the Maxwell's time-harmonic wave equation for the electric or for the magnetic fields both give the same results within the numerical error ranges (which are similar for both methods). When the magnetic/electric field is computed directly, the electric/magnetic field necessary for fluxcomputation is obtained from numerical differentiation of the directly computed field. This in principle could be subject to additional numerical errors. Therefore we have also performed simulation runs where we performed independent computations of both, electric and magnetic near fields and computed the electromagnetic flux through the detector from both of them. Also here we got the same quantitative results for $S / S_{0}$ and comparable magnitude of $\Delta\left(S / S_{0}\right)_{\text {rel }}$. Also, rounding of the computational domain has no significant influence. Further, numerical parameters for the transparent boundary setting are obtained automatically and are different for each computation which makes influence of these parameters on the results beyond computed error estimates very improbable.

To summarize, we observe convergence of the results towards a value of $S / S_{0} \approx 2.198825944 \pm 0.000000002$. We observe convergence towards this value under increase of the polynomial degree of the used FEM ansatz functions. We also observe convergence towards the same value when the mesh is refined, where adaptive mesh
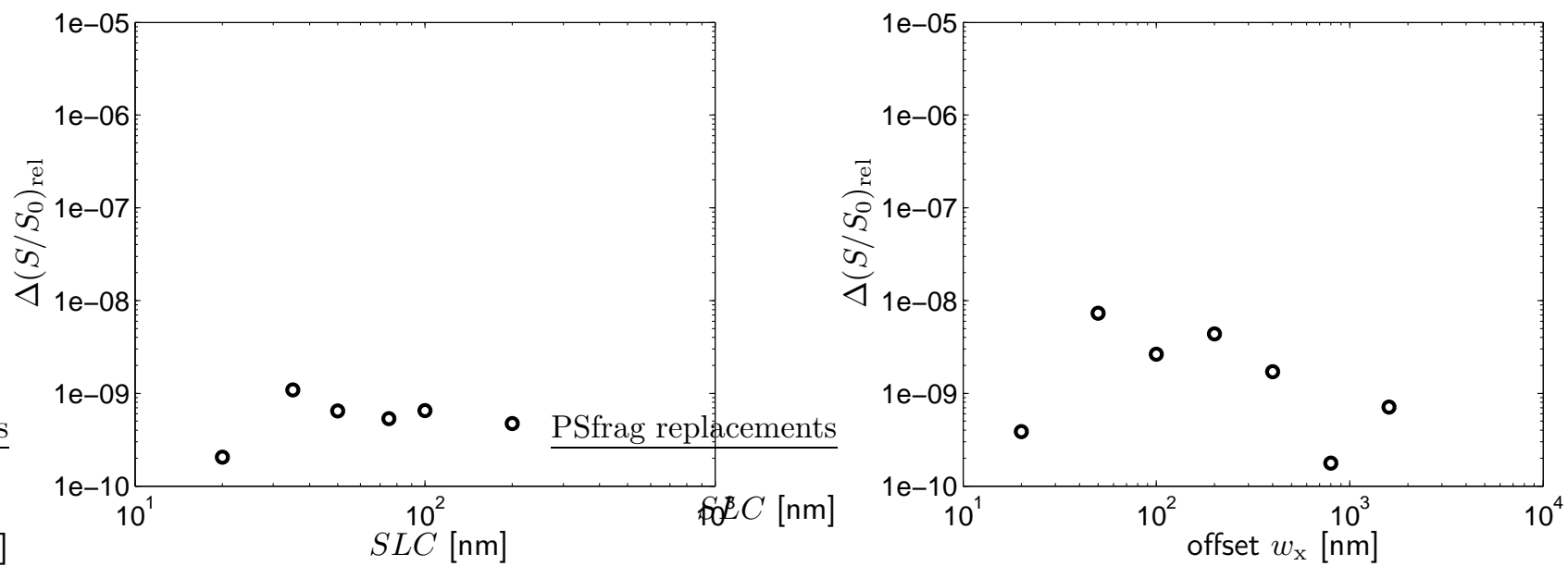

Figure 4. Left: Relative numerical error, $\Delta\left(S / S_{0}\right)_{\text {rel }}$, for various settings of initial mesh sidelength constraints, $S L C$, and otherwise fixed numerical parameters. Right: Relative numerical error, $\Delta\left(S / S_{0}\right)_{\text {rel }}$, for various settings of offset of the computational domain boundary from the scattering structures, offset $w_{\mathrm{x}}$, and otherwise fixed numerical parameters. As expected, the numerical error is dominated by the fixed numerical parameters. This shows that major contribution of $S L C$ and offset $w_{\mathrm{x}}$ on the numerical error budget can be excluded. Please, compare also Table 3 (data sets V and VI). 
refinement using a local error estimator, or (alternatively) using prerefinement of the mesh around corners in the geometry is used. Also global mesh refinement shows convergence towards the same value. We observe neither influence of PML parameters nor influence of placement of the computational domain boundary on the numerical results beyond those error limits which can be explained by the discretization parameters mesh refinement and FEM degree. Computation times for reaching relative accuracies of a level of $\Delta\left(S / S_{0}\right)_{\text {rel }} \approx 10^{-6}$ are in the range of few seconds on a standard PC. Significantly better performance by orders of magnitude in comparison to the finite-element methods discussed in the literature ${ }^{12}$ can be explained (i) by the usage of higher-order edge-elements ( $p=2$ is used in ${ }^{12}$ ), (ii) by usage of adaptive mesh refinement using automatic error-estimation (local refinement along metal-dielectric interfaces is used by FEM1 in, $\frac{12}{2}$ which is in contrast to error-estimation based automatic local mesh refinement which refines towards corners, regular meshes are used by FEM2 in 12 ), (iii) possibly by the specific implementation of transparent boundary conditions.

\section{CONCLUSION}

Numerical convergence results from a Maxwell-solver based on a finite-element method have been presented for a challenging benchmark problem. The benchmark case treats rigorous light scattering in a non-periodic setup. Numerical performance and obtained accuracy are improved by several orders of magnitude when compared to results from the literature for this specific benchmark case. Fields of application of the method are, e.g., in scatterometric and ellipsometric metrology setups as well as in computational lithography. Highly efficient numerical performance is required in these fields for current and future technology node applications.

\section{Acknowledgments}

The work presented here is part of the EMRP Joint Research Project IND 17 ScATTEROMETRY. The EMRP is jointly funded by the EMRP participating countries within EURAMET and the European Union. We further acknowledge German research foundation, DFG, for funding within DFG research center MATHEON, project D23: Design of nanophotonic devices and materials.

\section{REFERENCES}

[1] Lorenz, J. et al., "ITRS - International Technology Roadmap for Semiconductors, Modeling and Simulation Working Group," (2012). ITRS Winter Conference, http://www.itrs.net.

[2] Lai, K., "Review of computational lithography modeling: focusing on extending optical lithography and design-technology co-optimization," Adv. Opt. Techn. 1, 249 (2012).

[3] Pang, L., Peng, D., Hu, P., Chen, D., He, L., Li, Y., Satake, M., and Tolani, V., "Computational metrology and inspection (CMI) in mask inspection, metrology, review, and repair," Adv. Opt. Techn. 1, 299 (2012).

[4] Burger, S., Köhle, R., Zschiedrich, L., Gao, W., Schmidt, F., März, R., and Nölscher, C., "Benchmark of FEM, waveguide and FDTD algorithms for rigorous mask simulation," Proc. SPIE 5992, 599216 (2005).

[5] Pomplun, J., Burger, S., Zschiedrich, L., and Schmidt, F., "Adaptive finite element method for simulation of optical nano structures," phys. stat. sol. (b) 244, 3419 (2007).

[6] Burger, S., Zschiedrich, L., Pomplun, J., Blome, M., and Schmidt, F., "Advanced finite-element methods for design and analysis of nano-optical structures: Applications," Proc. SPIE 8642, 864205 (2013).

[7] Burger, S., Zschiedrich, L., Schmidt, F., Köhle, R., Henkel, T., Küchler, B., and Nölscher, C., "3D simulations of electromagnetic fields in nanostructures," Proc. SPIE 6617, 6617OV (2007).

[8] Pomplun, J., Burger, S., Schmidt, F., Scholze, F., Laubis, C., and Dersch, U., "Metrology of EUV masks by EUV-scatterometry and finite element analysis," Proc. SPIE 7028, 70280P (2008).

[9] Pomplun, J., Zschiedrich, L., Burger, S., Schmidt, F., Tyminski, J., Flagello, D., and Toshiharu, N., "Reduced basis method for source mask optimization," Proc. SPIE 7823, 78230E (2010).

[10] Burger, S., Zschiedrich, L., Pomplun, J., Schmidt, F., Kato, A., Laubis, C., and Scholze, F., "Investigation of 3D patterns on EUV masks by means of scatterometry and comparison to numerical simulations," Proc. SPIE 8166, 81661Q (2011). 
[11] Kleemann, B. H., Kurz, J., Hetzler, J., Pomplun, J., Burger, S., Zschiedrich, L., and Schmidt, F., "Fast online inverse scattering with Reduced Basis Method (RBM) for a 3D phase grating with specific line roughness," Proc. SPIE 8083, 808309 (2011).

[12] Lalanne, P., Besbes, M., Hugonin, J. P., van Haver, S., Janssen, O. T. A., Nugrowati, A. M., Xu, M., Pereira, S. F., Urbach, H. P., van de Nes, A. S., Bienstman, P., Granet, G., Moreau, A., Helfert, S., Sukharev, M., Seideman, T., Baida, F., Guizal, B., and van Labeke, D., "Numerical analysis of a slit-groove diffraction problem," Journal of the European Optical Society - Rapid publications 2, 07022 (2007).

[13] Burger, S., Zschiedrich, L., Schmidt, F., Köhle, R., Küchler, B., and Nölscher, C., "EMF simulations of isolated and periodic 3D photomask patterns," Proc. SPIE 6730, 67301W (2007).

[14] Burger, S., Zschiedrich, L., Schmidt, F., Evanschitzky, P., and Erdmann, A., "Benchmark of rigorous methods for electromagnetic field simulation," Proc. SPIE 7122, 71221S (2008).

[15] Hoffmann, J., Hafner, C., Leidenberger, P., Hesselbarth, J., and Burger, S., "Comparison of electromagnetic field solvers for the 3D analysis of plasmonic nano antennas," Proc. SPIE 7390, 73900J (2009).

[16] Maes, B., Petráček, J., Burger, S., Kwiecien, P., Luksch, J., and Richter, I., "Simulations of high-Q optical nanocavities with a gradual 1D bandgap," Opt. Express 21, 6794 (2013).

[17] Gay, G., Alloschery, O., De Lesegno, B. V., Odwyer, C., Weiner, J., and Lezec, H., "The optical response of nanostructured surfaces and the composite diffracted evanescent wave model," Nature Physics 2(4), 262-267 (2006).

[18] Zschiedrich, L., Klose, R., Schädle, A., and Schmidt, F., "A new finite element realization of the Perfectly Matched Layer Method for Helmholtz scattering problems on polygonal domains in 2D," J. Comput. Appl. Math. 188, 12 (2006).

[19] Zschiedrich, L., Burger, S., Pomplun, J., and Schmidt, F., "Goal oriented adaptive finite element method for the precise simulation of optical components," Proc. SPIE 6475, 64750H (2007).

[20] Zschiedrich, L., Burger, S., Schädle, A., and Schmidt, F., "A rigorous finite-element domain decomposition method for electromagnetic near field simulations," Proc. SPIE 6924, 692450 (2008).

\section{Appendix}

The numerical results of this benchmark case using FEM solver JCMsuite are summarized in Table 3. The table holds seven data sets: the numerical data setting for the quasi-exact result (see above), and for the parameter studies where each of the six numerical parameters has been varied independently. Results from data sets I to VI are displayed in Figures 2, 3, 4. The results from the literature are summarized in Table 2, where the relative errors have been estimated from the convergence graphs given in the publication. 12

\begin{tabular}{|l|l|l|l|}
\hline method & & $S / S_{0}$ & est. int. error \\
\hline MM1 & Aperiodic Fourier Modal Method (RCWA) & 2.206775 & \pm 0.004 \\
MM2 & Aperiodic Fourier Modal Method (RCWA) & 2.200904 & \pm 0.0001 \\
MM3 & Aperiodic Fourier Modal Method (RCWA) & 2.200952 & \pm 0.000004 \\
MM4 & Method of Lines (MOL) & 2.201970 & \pm 0.0002 \\
MM5 & Local Eigenmode-Modal method & 2.008785 & \pm 0.002 \\
FDTD1 & Finite-Difference Time-Domain Method & 2.211482 & \pm 0.01 \\
FDTD3 & Finite-Difference Time-Domain Method & 2.193380 & \pm 0.04 \\
FEM1 & Finite-Element Method & 2.201632 & \pm 0.001 \\
FEM2 & Finite-Element Method & 2.201143 & \pm 0.000006 \\
VIM & Volume Integral Method & 2.204575 & \pm 0.002 \\
HYB & Hybrid FEM-Modal Method & 2.200940 & \pm 0.00002 \\
\hline \hline FEM & Finite-Element Method (this paper) & 2.198826 & $\pm 1 \mathrm{e}-10$ \\
\hline
\end{tabular}

Table 2. Results on $S / S_{0}$ and relative numerical error (with respect to best converged solution of each respective method) estimated from Fig. 5 in Lalanne et al ${ }^{[12}$ For comparison, the last line of the table holds the quasi exact result from this paper. 


\begin{tabular}{|c|c|c|c|c|c|c|c|c|c|}
\hline data set & $N$ & $S / S_{0}$ & $\Delta\left(S / S_{0}\right)_{\mathrm{rel}}$ & $p$ & $n_{r}$ & $n_{a}$ & $d x[\mathrm{~nm}]$ & $S L C[\mathrm{~nm}]$ & offset [nm] \\
\hline Quasi-exact & 1026290 & 2.1988259440 & & 9 & 0 & 3 & 0.0010 & 50 & 200 \\
\hline \multirow[t]{11}{*}{ I } & 123282 & $\mathbf{2 . 1 9 8 8 3 4 3 7 2 2}$ & $3.83 \mathrm{e}-06$ & 4 & 0 & $\overline{0}$ & 0.2000 & 50 & 200 \\
\hline & 159612 & $\mathbf{2 . 1 9 8 8 2 9 9 6 6 7}$ & $1.83 \mathrm{e}-06$ & 4 & 0 & 1 & 0.2000 & 50 & 200 \\
\hline & 174806 & $\mathbf{2 . 1 9 8 8 2 7 8 1 1 0}$ & $8.49 \mathrm{e}-07$ & 4 & 0 & 2 & 0.2000 & 50 & 200 \\
\hline & 195658 & 2.1988268085 & $3.93 \mathrm{e}-07$ & 4 & 0 & 3 & 0.2000 & 50 & 200 \\
\hline & 221894 & $\mathbf{2 . 1 9 8 8 2 6 3 4 5 3}$ & $1.83 \mathrm{e}-07$ & 4 & 0 & 4 & 0.2000 & 50 & 200 \\
\hline & 250338 & $\mathbf{2 . 1 9 8 8 2 6 1 2 1 7}$ & $8.08 \mathrm{e}-08$ & 4 & 0 & 5 & 0.2000 & 50 & 200 \\
\hline & 286414 & $\mathbf{2 . 1 9 8 8 2 6 0 2 2 2}$ & $3.56 \mathrm{e}-08$ & 4 & 0 & 6 & 0.2000 & 50 & 200 \\
\hline & 329320 & $\mathbf{2 . 1 9 8 8 2 5 9 5 9 9}$ & $7.27 \mathrm{e}-09$ & 4 & 0 & 7 & 0.2000 & 50 & 200 \\
\hline & 384652 & $\mathbf{2 . 1 9 8 8 2 5 9 1 6 8}$ & $1.23 \mathrm{e}-08$ & 4 & 0 & 8 & 0.2000 & 50 & 200 \\
\hline & 442726 & $\mathbf{2 . 1 9 8 8 2 5 9 0 6 8}$ & $1.69 \mathrm{e}-08$ & 4 & 0 & 9 & 0.2000 & 50 & 200 \\
\hline & 527282 & 2.1988259319 & $5.51 \mathrm{e}-09$ & 4 & 0 & 10 & 0.2000 & 50 & 200 \\
\hline \multirow[t]{6}{*}{ II } & 157184 & $\mathbf{2 . 1 9 8 8 2 5 5 5 0 6}$ & $1.79 \mathrm{e}-07$ & 4 & 0 & 0 & 0.0100 & 50 & 200 \\
\hline & 622286 & $\mathbf{2 . 1 9 8 8 2 5 6 7 0 2}$ & $1.25 \mathrm{e}-07$ & 4 & 1 & 0 & 0.0100 & 50 & 200 \\
\hline & 2308698 & $\mathbf{2 . 1 9 8 8 2 5 8 4 9 8}$ & $4.28 \mathrm{e}-08$ & 4 & 2 & 0 & 0.0100 & 50 & 200 \\
\hline & 8489394 & $\mathbf{2 . 1 9 8 8 2 5 9 0 0 6}$ & $1.97 \mathrm{e}-08$ & 4 & 3 & 0 & 0.0100 & 50 & 200 \\
\hline & 33140834 & $\mathbf{2 . 1 9 8 8 2 5 9 2 3 5}$ & $9.32 \mathrm{e}-09$ & 4 & 4 & 0 & 0.0100 & 50 & 200 \\
\hline & 125886658 & 2.1988259315 & $5.69 \mathrm{e}-09$ & 4 & 5 & 0 & 0.0100 & 50 & 200 \\
\hline \multirow[t]{7}{*}{ III } & 82756 & $\mathbf{2 . 1 9 7 8 7 0 7 2 3 5}$ & $4.34 \mathrm{e}-04$ & $\mathbf{1}$ & 0 & 3 & 0.0010 & 50 & 200 \\
\hline & 362394 & 2.1988182593 & $3.49 \mathrm{e}-06$ & 2 & 0 & 3 & 0.0010 & 50 & 200 \\
\hline & 783836 & $\mathbf{2 . 1 9 8 8 2 5 9 8 8 0}$ & $2.00 \mathrm{e}-08$ & 3 & 0 & 3 & 0.0010 & 50 & 200 \\
\hline & 542996 & $\mathbf{2 . 1 9 8 8 2 5 9 1 0 7}$ & $1.51 \mathrm{e}-08$ & 4 & 0 & 3 & 0.0010 & 50 & 200 \\
\hline & 432247 & 2.1988259442 & $8.61 \mathrm{e}-11$ & 5 & 0 & 3 & 0.0010 & 50 & 200 \\
\hline & 476798 & 2.1988259442 & $1.13 \mathrm{e}-10$ & 6 & 0 & 3 & 0.0010 & 50 & 200 \\
\hline & 637212 & 2.1988259440 & $2.43 \mathrm{e}-11$ & 7 & 0 & 3 & 0.0010 & 50 & 200 \\
\hline \multirow[t]{13}{*}{ IV } & 8825980 & $\mathbf{2 . 1 9 8 8 2 0 6 5 4 0}$ & $2.41 \mathrm{e}-06$ & 7 & 3 & 2 & 5.0000 & 50 & 200 \\
\hline & 10121834 & $\mathbf{2 . 1 9 8 8 2 7 0 8 0 8}$ & $5.17 \mathrm{e}-07$ & 7 & 3 & 2 & 2.0000 & 50 & 200 \\
\hline & 11749026 & $\mathbf{2 . 1 9 8 8 2 6 6 8 9 3}$ & $3.39 \mathrm{e}-07$ & 7 & 3 & 2 & 1.0000 & 50 & 200 \\
\hline & 13364822 & $\mathbf{2 . 1 9 8 8 2 6 0 2 3 8}$ & $3.63 \mathrm{e}-08$ & 7 & 3 & 2 & 0.5000 & 50 & 200 \\
\hline & 17065246 & $\mathbf{2 . 1 9 8 8 2 6 0 0 5 3}$ & $2.79 \mathrm{e}-08$ & 7 & 3 & 2 & 0.2000 & 50 & 200 \\
\hline & 19191720 & $\mathbf{2 . 1 9 8 8 2 5 9 8 7 5}$ & $1.98 \mathrm{e}-08$ & 7 & 3 & 2 & 0.1000 & 50 & 200 \\
\hline & 21128326 & $\mathbf{2 . 1 9 8 8 2 5 9 8 4 4}$ & $1.84 \mathrm{e}-08$ & 7 & 3 & 2 & 0.0500 & 50 & 200 \\
\hline & 22332186 & $\mathbf{2 . 1 9 8 8 2 5 9 3 3 2}$ & $4.89 \mathrm{e}-09$ & 7 & 3 & 2 & 0.0200 & 50 & 200 \\
\hline & 23847490 & 2.1988259410 & $1.35 \mathrm{e}-09$ & 7 & 3 & 2 & 0.0100 & 50 & 200 \\
\hline & 25328200 & 2.1988259432 & $3.46 \mathrm{e}-10$ & 7 & 3 & 2 & 0.0050 & 50 & 200 \\
\hline & 28455268 & 2.1988259448 & $3.95 \mathrm{e}-10$ & 7 & 3 & 2 & 0.0020 & 50 & 200 \\
\hline & 30191072 & 2.1988259449 & $4.20 \mathrm{e}-10$ & 7 & 3 & 2 & 0.0010 & 50 & 200 \\
\hline & 30826532 & 2.1988259443 & $1.72 \mathrm{e}-10$ & 7 & 3 & 2 & 0.0005 & 50 & 200 \\
\hline \multirow[t]{6}{*}{$\overline{\mathrm{V}}$} & 24405586 & 2.1988259450 & $4.73 \mathrm{e}-10$ & 7 & 3 & 0 & 0.0010 & 200 & 200 \\
\hline & 26543078 & 2.1988259454 & $6.54 \mathrm{e}-10$ & 7 & 3 & 0 & 0.0010 & 100 & 200 \\
\hline & 28017866 & 2.1988259451 & $5.32 \mathrm{e}-10$ & 7 & 3 & 0 & 0.0010 & 75 & 200 \\
\hline & 30172354 & 2.1988259454 & $6.45 \mathrm{e}-10$ & 7 & 3 & 0 & 0.0010 & 50 & 200 \\
\hline & 36002178 & $\mathbf{2 . 1 9 8 8 2 5 9 4 6 3}$ & $1.08 \mathrm{e}-09$ & 7 & 3 & 0 & 0.0010 & 35 & 200 \\
\hline & 58449498 & 2.1988259444 & $2.05 \mathrm{e}-10$ & 7 & 3 & 0 & 0.0010 & 20 & 200 \\
\hline \multirow[t]{7}{*}{ VI } & 8408962 & 2.1988259448 & $3.87 \mathrm{e}-10$ & 4 & 3 & 2 & 0.0100 & 50 & 20 \\
\hline & 8278130 & $\mathbf{2 . 1 9 8 8 2 5 9 6 0 0}$ & $7.31 \mathrm{e}-09$ & 4 & 3 & 2 & 0.0100 & 50 & 50 \\
\hline & 7565714 & 2.1988259498 & $2.64 \mathrm{e}-09$ & 4 & 3 & 2 & 0.0100 & 50 & 100 \\
\hline & 8499474 & $\mathbf{2 . 1 9 8 8 2 5 9 3 4 3}$ & $4.39 \mathrm{e}-09$ & 4 & 3 & 2 & 0.0100 & 50 & 200 \\
\hline & 10140418 & $\mathbf{2 . 1 9 8 8 2 5 9 4 0 2}$ & $1.71 \mathrm{e}-09$ & 4 & 3 & 2 & 0.0100 & 50 & 400 \\
\hline & 14758242 & 2.1988259436 & $1.76 \mathrm{e}-10$ & 4 & 3 & 2 & 0.0100 & 50 & 800 \\
\hline & 27815378 & $\mathbf{2 . 1 9 8 8 2 5 9 4 2 4}$ & $7.08 \mathrm{e}-10$ & 4 & 3 & 2 & 0.0100 & 50 & 1600 \\
\hline
\end{tabular}

Table 3. Convergence study: FEM results for different numerical parameter settings. Number of unknowns, $N$, normalized energy flux, $S / S_{0}$, relative deviation from the quasi-exact result, $\Delta\left(S / S_{0}\right)_{\text {rel }}$, FEM degree $p$, number of regular and adaptive mesh refinement steps, $n_{r}$ and $n_{a}$, mesh prerefinement at corners, $d x$, mesh sidelength constraint, $S L C$, computational domain boundary offset. 